\title{
Brainstem encephalitis and the Miller Fisher syndrome
}

\author{
R K H Petty, R Duncan, G A Jamal, D Hadley, P G E Kennedy
}

\begin{abstract}
A case is described of a man with an acute onset areflexic ataxic syndrome with partial ophthalmoparesis and normal limb muscle power. There was evidence of both a peripheral neuropathy and a brainstem encephalitis. The patient made a full recovery and the interrelationship and nosology of brainstem encephalitis and the Miller Fisher syndrome are discussed.
\end{abstract}

(F Neurol Neurosurg Psychiatry 1993;56:201-203)

The possible relationship between the Miller Fisher (MFS) and Guillain-Barré syndromes (GBS) has been the subject of much debate..$^{1-3}$ Some authors have regarded MFS as a variant of $\mathrm{GBS}^{4}$ while others have felt the MFS involves the brainstem and bears similarities to the brainstem encephalitis of Bickerstaff (BBE). ${ }^{5}$ We report a patient in whom the clinical features and investigations were consistent with both an acute cranial and limb polyneuropathy and brainstem encephalitis and discuss the nosology of BBE and MFS.

\section{Case report}

A 32 year old man with no past medical or family history, and no history of alcohol abuse was admitted with a four day history of occipital headache and subsequent numbness of the right cheek, nausea and vomiting, diplopia and unsteadiness. Examination revealed phasic nystagmus on right and left gaze, impaired pinprick sensation in the distribution of the right trigeminal nerve and right limb ataxia. Reflexes were intact. CSF contained 229 white cells $\mathrm{Ml}$ (90\% lymphocytes, $10 \%$ polymorphonuclear cells), and protein of $0.32 \mathrm{G} / \mathrm{l}$. The next day he developed slurred dysarthria, hoarseness and nasal regurgitation. $\mathrm{He}$ had a partial right abducens palsy, with phasic nystagmus on lateral and upgaze. There was sensory loss in trigeminal territory on the right, with right lower motor neuron facial weakness and right palatal palsy. He had normal limb power and tone but reflexes were absent, except for the right biceps and brachioradialis. There was mild symmetrical limb ataxia and dysequilibrium so that he required assistance to walk. Sensation was normal.

Cranial CT was normal. MRI (fig) showed lesions in the posterolateral brainstem ped- uncular area on either side of the fourth ventricle, the right side being larger and longer. These lesions were hyperintense on $T_{2}$ weighted and hypointense on $T_{1}$ weighted images. After intravenous injection of Dimeglumine gadopentetate both lesions showed $T_{1}$ shortening, confirming blood-brain barrier breakdown. Neurophysiological studies demonstrated normal peripheral motor nerve conduction and sensory potentials. $F$ waves were absent in both peroneal and right median nerves (table). H waves were absent in both tibial nerves. EMG of proximal and distal lower limb muscles showed no evidence of denervation.

He developed bilateral facial weakness, severe limb ataxia, dysequilibrium and total areflexia, although he remained alert. He improved after treatment with high dose methylprednisolone and plasma exchange. Neurophysiological studies two weeks after presentation showed normal motor and sensory velocities, and an $\mathrm{F}$ wave now detectable on stimulation of the right median nerve. Electromyography showed evidence of denervation in the right masseter and orbicularis oculi muscles. Two weeks after plasma

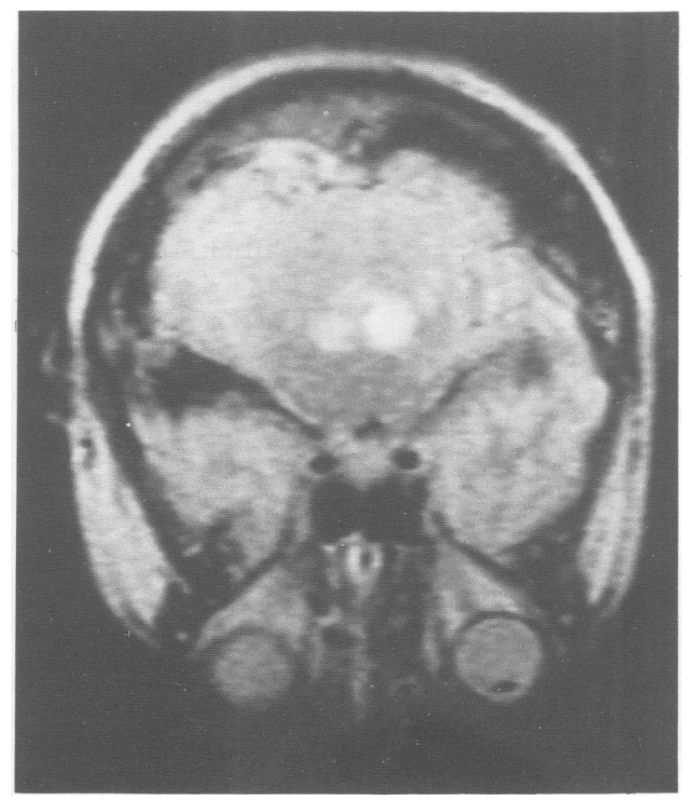

Figure Cranial MRI, $T_{2}$ weighted (SE 2000/80) axial section showing areas of hyperintensity in the posterolateral brain stem peduncular areas, on both sides of the fourth ventricle 
Table Neurophysiological studies

\begin{tabular}{lllll}
\hline \multicolumn{4}{c}{ Day of study } \\
\cline { 2 - 5 } & & Day 5 & Day 13 & Day 139 \\
\hline Common Peroneal & $\mathrm{m} / \mathrm{sec}$ & 50 & 53 & 53 \\
Motor velocity, & $\mathrm{mV}$ & $14 \cdot 4$ & $12 \cdot 4$ & 7 \\
Amplitude & $\mathrm{ms}$ & $3 \cdot 7$ & $4 \cdot 1$ & $3 \cdot 2$ \\
Latency & $\mathrm{ms}$ & $\mathrm{ND}$ & $\mathrm{ND}$ & 54 \\
F wave latency & & & & \\
Median & $\mathrm{m} / \mathrm{s}$ & 61 & 52 & - \\
Velocity, & $\mathrm{mV}$ & $7 \cdot 3$ & $7 \cdot 8$ & - \\
Amplitude & $\mathrm{ms}$ & $4 \cdot 0$ & $3 \cdot 8$ & - \\
Latency & $\mathrm{ms}$ & $\mathrm{ND}$ & 30 & 32 \\
F wave latency & & & & \\
Sural nerve & $\mu \mathrm{V}$ & 11 & $9 \cdot 3$ & $9 \cdot 8$ \\
Amplitude & $\mathrm{ms}$ & $2 \cdot 2$ & $3 \cdot 3$ & $3 \cdot 8$ \\
Latency & & & \\
\hline ND $=$ not detected. & &
\end{tabular}

exchange he was able to walk unaided, with a partial right abducens weakness, horizontal nystagmus on right lateral gaze and persisting facial weakness. His speech had improved, he had recovered his limb reflexes and had only minimal limb ataxia. Normal or negative investigations during the acute illness included EEG, visual evoked responses, chest radiograph, full blood count, ESR, serum electrolytes, syphilis serology, autoantibody screen, serology for borrelia, legionella, toxoplasma, HIV-I, brucella, hepatitis B, Epstein-Barr virus, mycoplasma, urinary porphyrin screen, and serum electrophoretic strip.

Six months later he had mild residual facial weakness, normal reflexes and no limb or gait ataxia. MRI showed resolution of the previous abnormalities, and $\mathrm{F}$ waves were now detectable on stimulation of both peroneal and median nerves.

\section{Discussion}

This patient suffered a monophasic illness with virtually complete recovery. Diagnoses of both MFS and BBE were considered but clinical and laboratory features suggested both intrinsic brainstem and peripheral nerve disease, contrary to definitions of both these disorders.

Bickerstaff ${ }^{2}$ reported eight cases of "brainstem encephalitis". All presented with drowsiness and a bulbar paresis including ophthalmoparesis. Of these eight, six developed deafness, six were ataxic, five had depressed or absent reflexes and three extensor plantars. During recovery six showed undue lability of emotions and three showed Parkinsonian features. Six patients had a CSF pleocytosis. These cases were notable for recovery despite severe neurological deficit, the severe cranial nerve dysfunction without respiratory or cardiovascular disturbance and the neuropsychiatric and extrapyramidal features occurring during recovery.

Subsequent cases described as BBE have not fulfilled these criteria. Al-Din et al ${ }^{5}$ included cases with a cranial polyneuropathy and ataxia with areflexia and an elevated CSF protein (for example, their cases $3,5,13,15,16,18$ ). None showed late psychiatric or extrapyramidal features. The cases reported as BBE by Yaqub et $a l^{6}$ did not show neuropsychiatric features during recovery although they did show features of a brainstem disorder and MRI changes similar to our case. We are not aware of recently described cases fulfilling all the diagnostic criteria laid down by Bickerstaff.

In 1956 Miller Fisher ${ }^{1}$ described three cases which he regarded as variants of "acute idiopathic polyneuritis". All patients had internal and external ophthalmoplegic, ataxia and areflexia. Late neuropsychological features were absent. CSF protein was elevated in one patient. He proposed that these cases were variants of GBS, citing cases of GBS with cranial nerve involvement including internal and external ophthalmoplegia. The symmetry of the ophthalmoparesis and the simulation of paralyses of conjugate gaze was unusual for a purely peripheral lesion. There is supportive evidence that the ataxia could result from mismatch of input of joint receptors and proprioception to the CNS. ${ }^{478}$

Thus there is a body of opinion that considers the MFS as purely a peripheral cranial polyneuropathy, yet in at least some patients in whom MRI was performed central involvement was documented. ${ }^{910}$ Similarly, detailed neurophysiological studies have not been carried out in cases labelled as brainstem encephalitis.

The interest in our case lies in its similarities to both these entities, and the implications for pathogenesis. There appears to be little doubt that our patient had an acute inflammatory brainstem disorder; MRI was abnormal and showed recovery, and clinical features were consistent with this. In addition, there were features of peripheral nerve disease; areflexia with recovery, although this has been reported in brainstem disease, ${ }^{11}$ nerve conduction studies showed initially absent and subsequently recovering $F$ waves; although velocities remained normal and the cause of the absent $F$ waves could not be clearly localised. This case cannot be accepted as MFS; there was only partial ophthalmoparesis, the CSF showed a prominent lymphocytosis, and the MRI showed clear central involvement.

There have been two previous reports of MRI abnormalities in cases resembling our own. ${ }^{9}{ }^{10}$ Taphoorn ${ }^{9}$ described CT and MRI abnormalities within the brainstem in a patient with features of both GBS and the MFS. Neurophysiological investigations were consistent with a demyelinating neuropathy. The patient apparently improved but no neurophysiological or MRI follow up was reported. Giroud $e t a l^{10}$ reported a similar patient with an areflexic ataxic syndrome whose MRI showed an area of high T2 signal in the pons. As in the previous case no follow up scan was reported.

This case exemplifies the problems with definition of both the MFS and BBE. It is possible that further MRI of patients with clinical features similar to the MFS may reveal other patients with evident central involvement and that full neurophysiological investigation of patients with brainstem encephalitis of whatever cause may identify a proportion of patients with peripheral involvement. In view 
of the well recognised lack of other brainstem features in these cases, this peripheral involvement may even be the dominant lesion.

1 Miller Fisher. An unusual variant of acute idopathic polyneurities (syndrome of opthalmoplegia, ataxia and polyneurities (syndrome of opthalmoplegia,

2 Bickerstaff ER. Brainstem encephalitis. Further observations on a grave syndrome with a benign prognosis. $B M \mathcal{F}$

3 Bickerstaff ER. Brainstem encephalitis (Bickerstaff's encephalitis). In: Vinken PJ, Bruyn GW, eds. Handbook of clinical neurology, vol 34. Amsterdam, North Holland, 1978:605-9.

4 Jamal GA, Ballantyne JP. The localisation of the lesion in patients with acute ophthalmoplegia, ataxia and areflexia (Miller Fisher syndrome). A serial multimodal neurophysiological study. Brain 1988;111:95-114.
5 Al-Din AN, Anderson M, Bickerstaff ER, Harvey I. Brainstem encephalitis and the syndrome of Miller Fishers. A clinical study. Brain 1982;105:481-95.

6 Yaqub BA, Al-Deeb SM, Daif AK, et al. Bickerstaff brainstem encephalitis. A grave non-demyelinating disease with a benign prognosis. $\mathcal{F}$ Neurological Sciences 1990;96:29-40.

7 Ropper AH, Shahani B. Proposed mechanism of ataxia in Fisher's syndrome. Arch Neurol 1983;40:537-8.

8 Jamal GA, Donaghy M. A peripheral mechanism for the ataxia associated with the Miller Fisher syndrome of acute ophthalmoplegia, ataxia and areflexia (Abstr). 7 Neurol Neurosurg Psychiatry 1989;52:1210.

9 Taphoorn MJB, Lanting P, Hazenberg GJ, Uitdehaag BJM, Kaiser MC. Observations on the lesion site in the Miller Fisher syndrome: presentation of a case including CT and MRI. Acta Neurol Scand 1989;80:167-9.

10 Giroud M, Mousson C, Chapolin JM, Rifle G, Dumas R. Miller Fisher syndrome and pontine abnormalities on MRI: a case report. $\mathcal{F}$ Neurol 1990;237:489-90.

11 Asbury AK, McKhann GM, McDonald WI. Diseases of the nervous system. London: Heinemann 1986:355-6. 